\title{
ARTICLE
}

\section{Uric acid pathway activation during respiratory virus infection promotes Th2 immune response via innate cytokine production and ILC2 accumulation}

Wendy Fonseca ${ }^{1}$, Carrie-Anne Malinczak ${ }^{1}$, Charles F. Schuler ${ }^{2}$, Shannon K. K. Best ${ }^{1}$, Andrew J. Rasky ${ }^{1}$, Susan B. Morris ${ }^{1}$, Tracy X. Cui ${ }^{4}$, Antonia P. Popova ${ }^{4}$ and Nicholas W. Lukacs ${ }^{1,3}$

Respiratory syncytial virus (RSV) infects a majority of infants and can cause severe disease leading to increased risk to develop asthma later in life. In the present studies we detected high levels of uric acid pathway components during RSV infection and examined whether they altered the pathogenesis of RSV infection. Inhibition of uric acid (UA) pathway activation during RSV infection in airway epithelial cells using XOI decreased the expression of IL-33, thymic stromal lymphopoietin (TSLP), and CCL2. In addition, treatment of RSV infected bone marrow-derived macrophages with XOI decreased production of IL-1 $\beta$. Thus, UA activation of different cell populations contributes different innate immune mediators that promote immunopathogenesis. When mice were treated with XOI or interleukin-1 receptor antagonist (IL1-ra) during RSV infection decreased pulmonary mucus was observed along with significantly reduced numbers of ILC2 and macrophages, accompanied by decreased IL-33 in bronchoalveolar lavage of the treated mice. These findings provide mechanistic insight into the development of RSV immunopathology and indicate that xanthine metabolites and UA are key immunoregulator molecules during RSV infection. Moreover, these findings suggest uric acid and IL-1 $\beta$ as possible therapeutic targets to attenuate severe RSV disease.

Mucosal Immunology (2020) 13:691-701; https://doi.org/10.1038/s41385-020-0264-z

\section{INTRODUCTION}

Respiratory syncytial virus (RSV) infects nearly all children by age 2 and is the leading cause of bronchiolitis in children worldwide. ${ }^{1,2}$ RSV preferentially infects airway epithelium, and it is especially detrimental in very young infants whose airways are small and easily occluded. ${ }^{3}$ RSV infection has been linked with the development of asthmatic airway disease later in childhood. ${ }^{4-6}$ Children hospitalized with RSV bronchiolitis were at higher risk of developing asthma and these kids experienced severe episodes of wheezing at ages 1,3 , and 7 years of age when compared to healthy controls. ${ }^{7,8}$ RSV-bronchiolitis typically is accompanied by airway epithelial cell destruction and sloughing, mucus hypersecretion, edema, peribronchiolar inflammatory infiltration, and pulmonary obstruction. ${ }^{9,10}$ Increased mucus production has been associated with the production of IL-13 and the development of goblet cell hyperplasia. ${ }^{11}$ An important source of IL-13 in the lungs is the group 2 innate lymphoid cells (ILC2s). ${ }^{12,13}$ The ILC2s are activated directly by epithelium-associated cytokines IL-25, IL-33, and TSLP. ${ }^{12}$ Previous studies have shown that RSV infection can induce IL-33 production and that this cytokine plays a critical role in the pathogenesis of the disease by activating ILC2s to produced IL-13 in the lung. ${ }^{14,15}$ It has also been shown that IL-1 $\beta$ is a critical activator of ILC2s cells, inducing proliferation and cytokine production and regulating the expression of epithelial cytokine receptors. ${ }^{16}$ Increased expression and production of $\mathrm{IL}-1 \beta$ during
RSV infection has also been reported through the activation of the inflammasome. ${ }^{17,18}$ Uric acid is a common danger associated molecular pattern (DAMP) molecule that is known to activate the inflammasome through NLRP3. ${ }^{19,20}$ There is a large literature and clinical studies have shown that inhibitors of uric acid are safe not only in mice but in humans, including infants, over a vast array of diseases that they have been used. ${ }^{21-25}$

Our previous studies have begun to investigate how RSV infection alters metabolite production and how particular metabolic pathways impact the immune responses. ${ }^{26}$ In these studies, we investigated uric acid changes during RSV infection that leads to a number of detrimental pathological immune responses that can be modulated using $\mathrm{XOI}$ and by blocking the effect of the downstream inflammasome mediated IL-1 $\beta$ with IL-1 receptor antagonist (IL-1ra).

\section{MATERIALS AND METHODS}

Mouse model

All experiments involving the use of animals were approved by the University of Michigan Institutional Animal Care \& Use Committee. Female BALB/c mice, 6-8 weeks of age, were purchased from The Jackson Laboratory (Bar Harbor, ME). All mice were maintained under standard pathogen-free conditions. Female Balb/c mice 6-7 weeks old were intratracheally infected

\footnotetext{
${ }^{1}$ Department of Pathology, University of Michigan, Ann Arbor, MI 48109, USA; ${ }^{2}$ Department of Internal Medicine, Division of Allergy and Clinical Immunology, University of

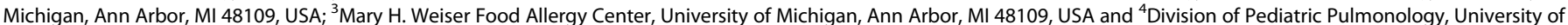
Michigan, Ann Arbor, Ml 48109, USA

Correspondence: Nicholas W. Lukacs (nlukacs@med.umich.edu)
}

Received: 5 July 2019 Revised: 5 January 2020 Accepted: 23 January 2020

Published online: 11 February 2020 
Respiratory Syncytial Virus (RSV) infection model

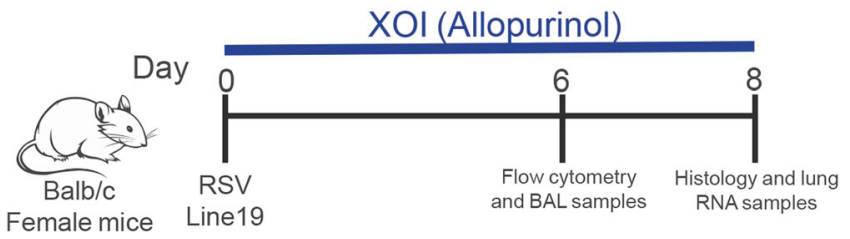

Fig. 1 Animal model. Female Balb/c mice 6-7 weeks old were intratracheally infected with RSV line $19(1 \times 105 \mathrm{pfu})$. The mice were treated with xanthine oxidase inhibitor (XOI) $(20 \mathrm{mg} / \mathrm{kg} / \mathrm{mouse}$ ) intraperitoneally or recombinant murine IL-1 ra $(100 \mu \mathrm{g} / \mathrm{kg} / \mathrm{mouse})$ intranasally every day during the time of infection.

with RSV $\left(1 \times 10^{5} \mathrm{pfu}\right)$ line 19 antigenic subgroup A. The mice were treated with Xanthine Oxidase inhibitor (XOI) $(20 \mathrm{mg} / \mathrm{kg} / \mathrm{mouse}$ ) intraperitoneally or with recombinant murine IL-1 ra (IL1 ra/100 $\mu \mathrm{g} /$ $\mathrm{kg} / \mathrm{mouse}$ ) intranasally every day during the time of infection. At day 6 post-infection samples were collected for flow cytometry and BAL to measure uric acid or cytokines, and at day 8 postinfection we collected samples for histology and RNA extraction (Fig. 1).

Respiratory syncytial virus

Our laboratory uses antigenic subgroup A, Line 19 RSV, obtained initially from a sick infant at the University of Michigan Hospital System. This isolate has been shown in animal models to mimic human infection by eliciting airway mucus production upon inoculation with $1 \times 10^{5}$ pfu RSV. ${ }^{27}$

Rhinovirus

RV1B (from American Type Culture Collection, Manassas, VA) were grown in HeLa cells, concentrated, partially purified, and titered as described. ${ }^{28}$ Viral titers were measured by plaque assay.

Human specimens

All human studies were performed in accordance with an approved University of Michigan institutional review board protocol after legal consent. The tracheal aspirate samples were diluted 50:50 with PBS containing complete anti-protease cocktail (Sigma-Aldrich, St. Louis, MO) and $0.5 \%$ Triton X-100 nonionic detergent to dissociate the mucus. Samples were aliquoted in 75 $\mu \mathrm{L}$ and stored at $-80^{\circ} \mathrm{C}$ until analysis. Tracheal aspirate samples were obtained from RSV infected children who were hospitalized and given mechanical ventilation in a pediatric intensive care unit. Control uninfected tracheal infant samples were obtained from children undergoing surgery for reasons unrelated to RSV infection, after parental consent. Uric acid levels were measured with a uric acid assay kit (Cayman Chemicals, USA), following manufacturer's instructions.

Quantitative RT-PCR

Lung tissue was homogenized in TRIzol reagent then RNA was extracted using TRIzol reagent (Invitrogen, Carlsbad, CA). CDNA was synthesized using murine leukemia virus reverse transcriptase (Applied Biosystems, Foster City, CA) and incubated at $37^{\circ} \mathrm{C}$ for 1 $\mathrm{h}$, followed by incubation at $95^{\circ} \mathrm{C}$ for $10 \mathrm{~min}$ to stop the reaction. Real-time quantitative PCR (qPCR) was multiplexed using TaqMan primers, with a FAM-conjugated probe and Gapdh with a VICconjugated probe (Applied Biosystems) to measure IL4, IL5, IL13, and Ifnb cytokines. Fold change was quantified using the $2^{-\Delta \Delta}$ cycle threshold (CT) method. Custom primers were designed to measure Muc5ac and Gob5 mRNA levels as described. ${ }^{29}$ All reactions were run on 7500 Real-Time PCR System (Applied Biosystems, Foster City, CA).

\section{Lung histology}

The left lung was perfused with $4 \%$ (vol/vol) formaldehyde for fixation and embedded in paraffin. Five-micrometer lung sections were stained with periodic acid-Schiff (PAS) to detect mucus production, and inflammatory infiltrates. Photomicrographs were captured using a Zeiss Axio Imager Z1 and AxioVision 4.8 software (Zeiss, Munich, Germany).

Enzyme-linked immunosorbent assays

Murine IL-1b and IL-33 proteins were quantified from bronchial alveolar lavages (BAL) samples taken from naïve or infected mice after euthanized. For IL-1b and IL-33, R\&D Duo set ELISA kit (R\&D Systems, Minneapolis, MN) was used following the manufacturer's instruction.

\section{Flow cytometry}

The lungs were removed, and single cells were isolated by enzymatic digestion with $1 \mathrm{mg} / \mathrm{ml}$ collagenase A (Roche, Indianapolis, IN) and 20U/ml DNase I (Sigma, St. Louis, MO) in RPMI 1640 containing $10 \%$ FCS. Tissues were further dispersed through an 18-gauge needle (10-ml syringe), RBCs were lysed, and samples were filtered through $100-\mu \mathrm{m}$ nylon mesh twice. Cells were resuspended in PBS and live cells were identified using LIVE/DEAD Fixable Yellow Dead Cell Stain kit (Thermo Fisher Scientific, Waltham, MA). Cells were resuspended in PBS with 1\% FCS and FC receptors were blocked with purified anti-CD16/32 (clone 93; BioLegend, San Diego, CA). Surface markers were identified using Abs (clones) against the following antigens, all from BioLegend: anti-Gr-1 (RB6- 8C5), B220 (RA3-6B2), CD3 (145-2C11), Ter119 (Ter119), CD11b (M1/70), CD25 (PC61), CD45 (30-F11), CD127 (SB/199), ST2 (DIH9), c-Kit (2B8), CD90 (53-2.1), CD4 (RM4-5), CD3 (17A2), CD8 (53-5.8), CD69 (H1.2F3) CD11C (N418), MHCII (M5/114.15.2), CD103 (2E7). SiglecF (E50-2440) was purchased from BD Biosciences (San Jose, CA). For innate lymphoid cell staining, lineage markers were anti-CD3, CD11b, B220, Gr-1, and TER119. ILC2: Lin-CD45+ CD90+ ST2+ c-Kit+ CD127+ GATA3+. Eosinophils: SSC high $C D 11 b+$ SiglecF + . Neutrophils: SSC ${ }^{\text {high }}$ CD11b+ SiglecF- GR-1+. Conventional DC: CD11b+ CD11c+ MHCIl+, CD103-. DC 103+: CD11c+ MHCll+ CD11b-CD103+. Interstitial macrophages: CD11b+CD11c-F4/80+. CD4+ T cells: CD3+ CD4+, CD8+T cells: $\mathrm{CD} 3+\mathrm{CD} 8+$.

Data was collected in a NovoCyte flow cytometer (ACEA Bioscience, Inc. San Diego, California). Data analysis was performed using FlowJo software (Tree Star, Oregon, USA).

\section{Protein lung extraction}

Lung protein was extracted using a tissue cell lysis reagent T-PER (Thermo Fisher), following manufacturer's instructions. The total protein was quantified using Pierce BCA assay kit (Thermo Fisher) and protein levels were measured with a Bio-Plex cytokine assay (Bio-Rad Laboratories).

Lund draining lymph nodes in vitro re-stimulated and cytokine production assay

Lund draining lymph nodes (LDLN) were enzymatically digested using $1 \mathrm{mg} / \mathrm{ml}$ collagenase A (Roche) and $25 \mathrm{U} / \mathrm{ml}$ DNase I (SigmaAldrich) in RPMI 1640 with $10 \%$ FCS for 45 min at $37^{\circ} \mathrm{C}$. Tissues were further dispersed through an 18-gauge needle (10-ml syringe). RBCs were lysed, and samples were filtered through $100-\mu \mathrm{m}$ nylon mesh twice. Cells $\left(5 \times 10^{5}\right)$ from LDLN cells were plated in 96-well plates and re-stimulated with RSV L19 MOI 1:1 for $48 \mathrm{~h}$. IFN- $\gamma$, IL-4, IL-5, IL-13, and IL-17a levels in supernatants were measured with a Bio-Plex cytokine assay (Bio-Rad Laboratories).

Uric acid measurements in BAL

Uric acid was measured from bronchoalveolar lavages samples taken from naïve or infected mice after euthanized at day 6 post- 
infection. We used the Uric acid assay kit (Cayman Chemicals, USA), and we followed manufacturer's instructions.

Mouse airway epithelial cells in primary culture

Whole lungs of naïve mice were digested in Dispase (BD Biosciences), filtered through the a $25-\mu \mathrm{m}$ mesh filter, and depleted of immune cells by labeling with biotinylated antibodies to CD16/32 and CD45 (BD Pharmingen), followed by labeling with anti-biotin microbeads and passage through a MACS column
(Miltenyi Biotec, Bergisch Gladbach, Germany). Depleted cell suspensions were adherence-purified overnight in DMEM-based complete media, and non-adherent cells cultured for 4 days in complete media within fibronectin-coated wells, yielding $\geq 90 \% \mathrm{E}$ cadherin positive cells as shown previously. ${ }^{17,30}$ The cells were maintained at $37^{\circ} \mathrm{C}, 5 \% \mathrm{CO}_{2}$. For the experiments, cells were seeded in 24 and 48 well plates at $70 \%$ confluency. Cultures were infected with RSV or Rhinovirus at 1:1 $\mathrm{MOI}$, for $24 \mathrm{~h}$ for RNA extraction. Prior to RSV infection, cells were treated with XOI (200 a

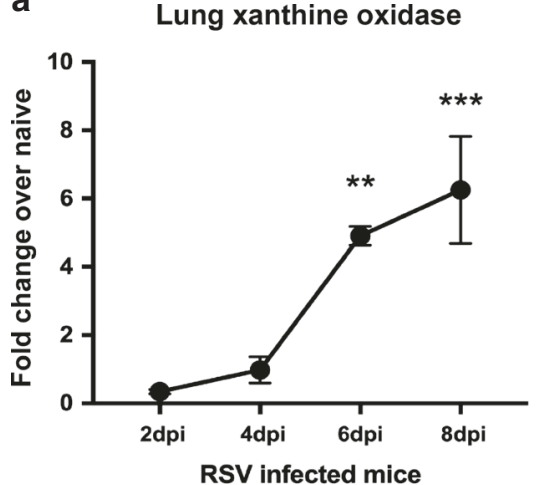

b

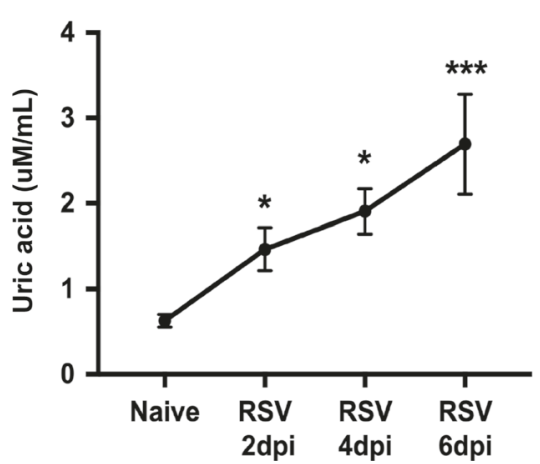

C

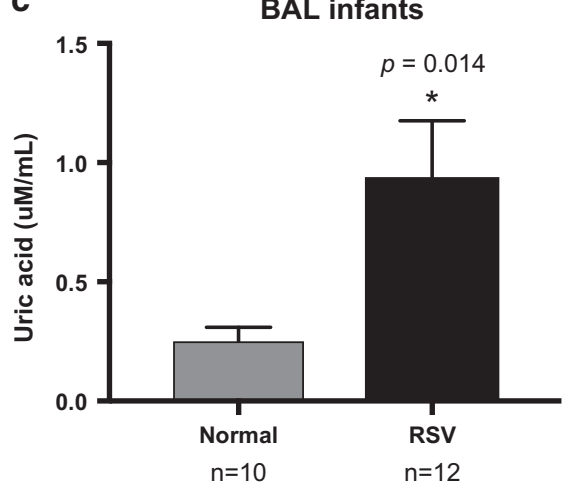

Fig. 2 RSV infection increases Uric acid production in the airways. a qPCR of Xanthine Oxidase (XO) in the lung of RSV infected mice shows significantly increased expression of XO during infection. $\mathbf{b}$ The uric acid levels in bronchoalveolar lavages (BAL) of RSV infected mice were significantly increased in the BAL of infected mice compared with the uric acid level in naive mice. Data represents the Mean \pm SE from 4 to 5 mice (experimental repeats $3-4) .{ }^{*} p \leq 0.05,{ }^{* *} p \leq 0.01,{ }^{* * *} p \leq 0.001$. c Uric acid levels from samples of lung aspirates from RSV + and normal infants, with significant upregulation in RSV+ infants. ${ }^{*} p \leq 0.05,{ }^{* *} p \leq 0.01$.

a

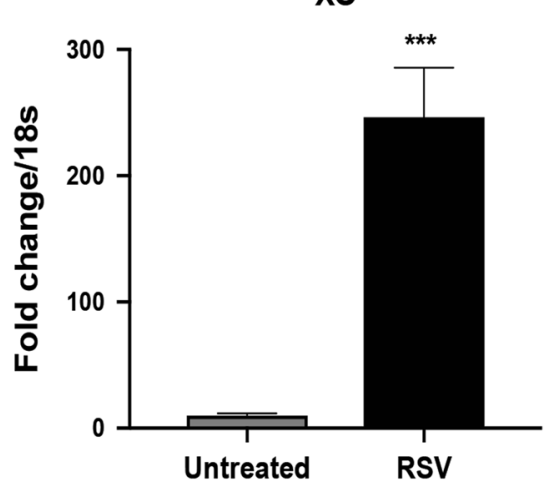

b

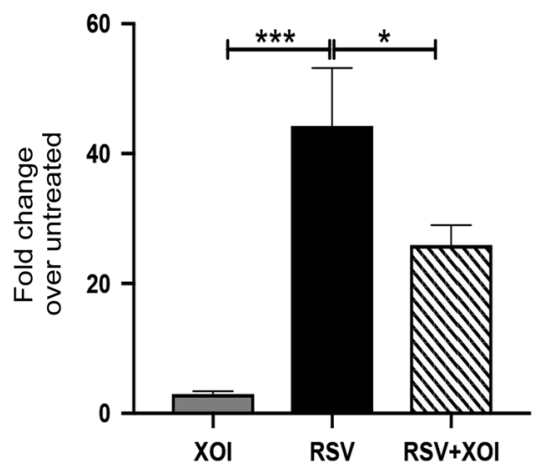

c

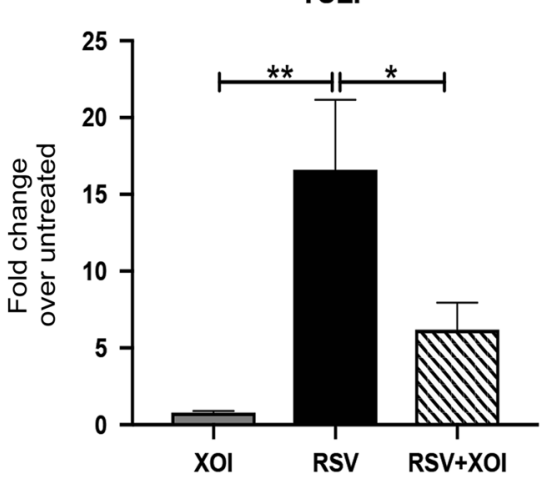

d
CCL2

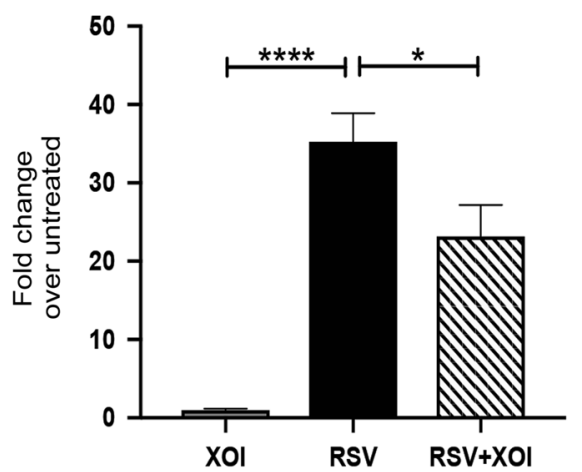

e

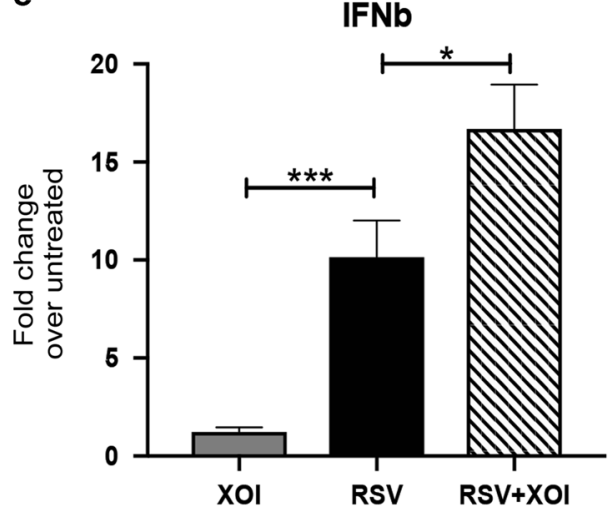

IFNb 
a

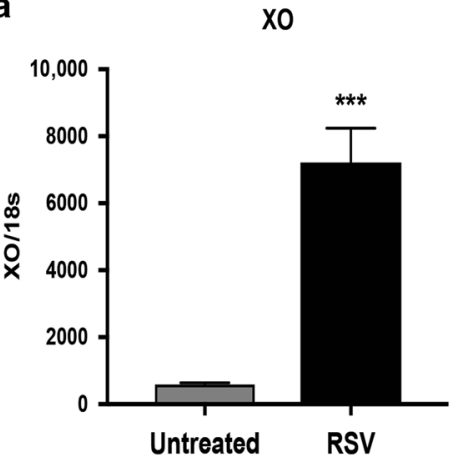

b

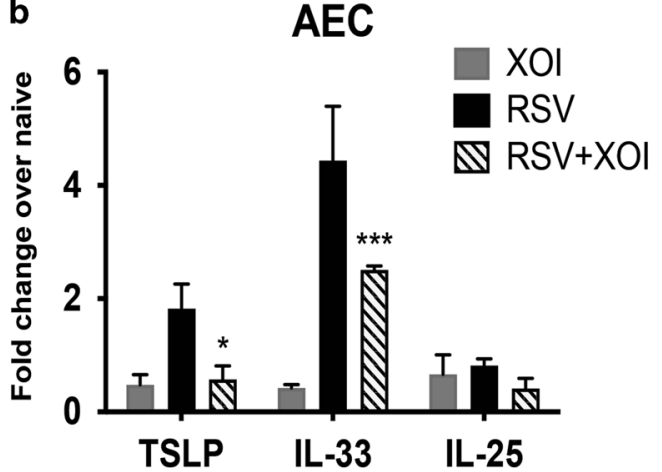

d

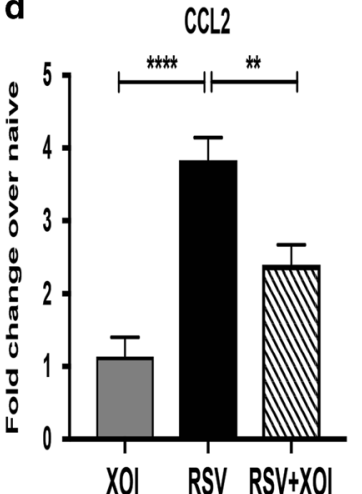

h

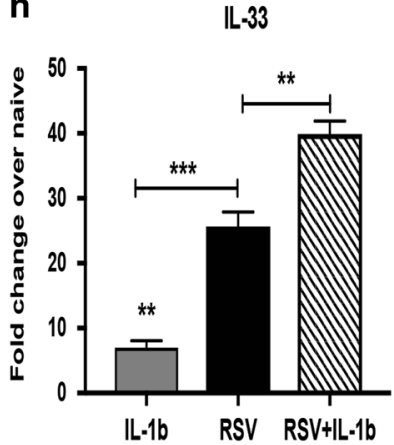

e

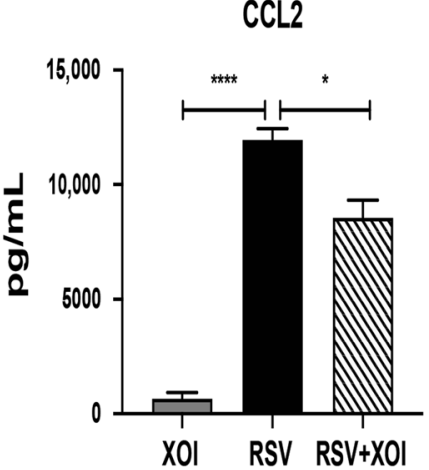

f
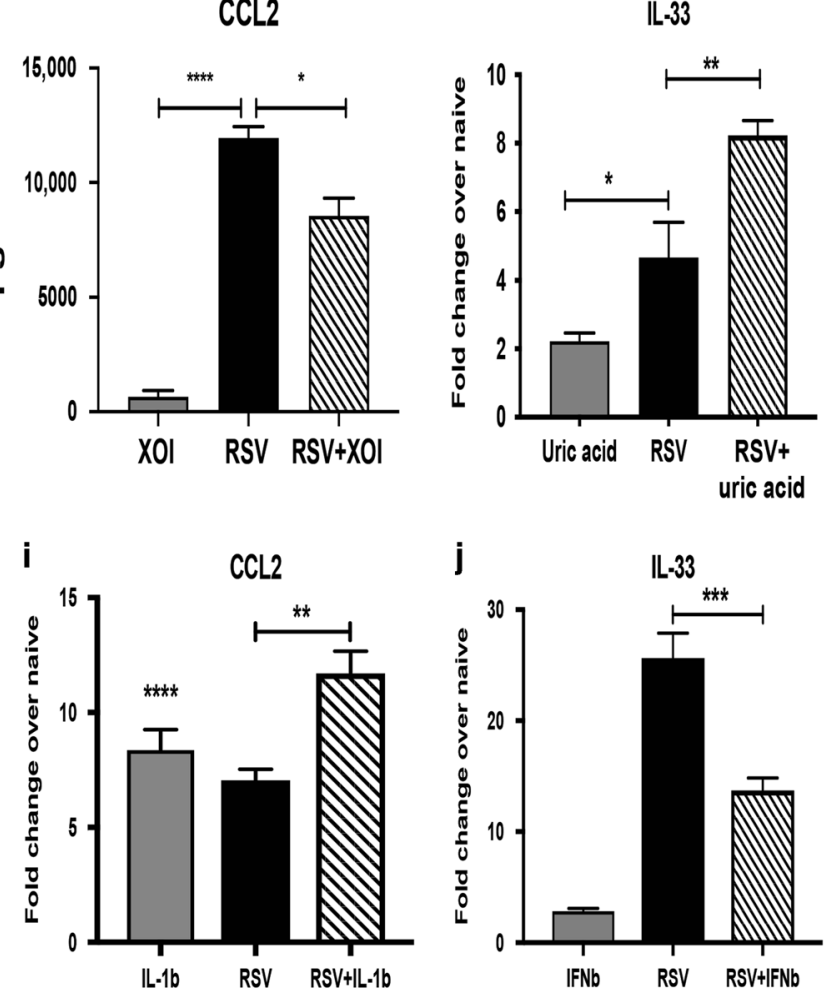

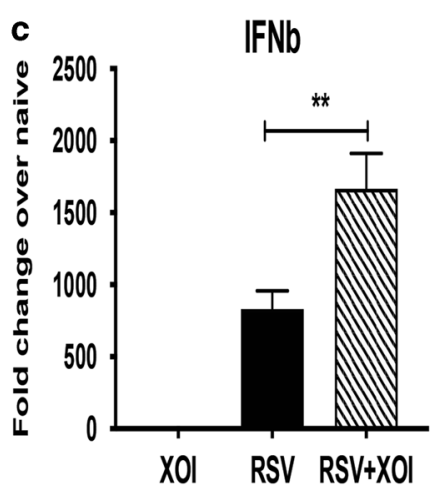

g

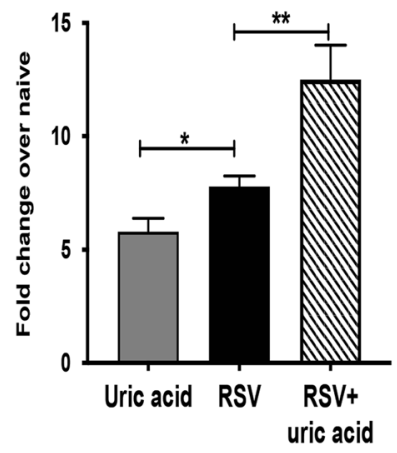

k

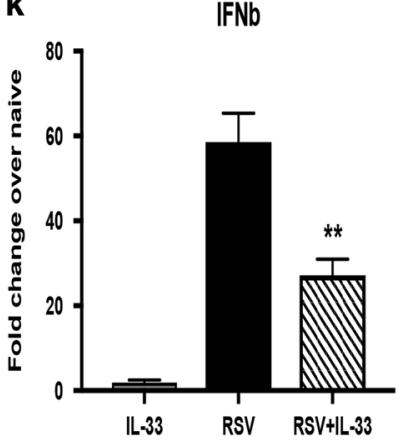

Fig. 4 Primary mouse airway epithelial cells increase the expression of XO during RSV infection and induce inflammatory innate cytokines. Cultures of primary airway epithelial cells (AECs) from naïve Balb/c mice were infected with RSV for $24 \mathrm{~h}$ and underwent different treatments. a Xanthine oxidase expression in RSV infected cells compared with uninfected cells. b, c TSLP, IL-33, and IL-25 and IFN $\beta$ expression in AECs infected with RSV treated with or without XOI. d, e CCL2 expression and production in in AECs infected with RSV treated with or without XOI. f, $\mathbf{g ~ I L - 3 3}$ and CCL2 expression in AECs that were treated with uric acid, RSV or RSV + uric acid. $\mathbf{h}, \mathbf{i}$ IL-33 and CCL2 expression in

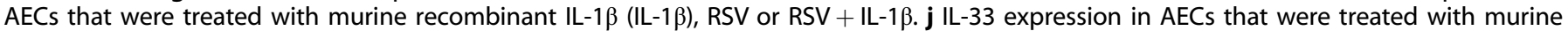
recombinant IFN $\beta$ (IFN $\beta$ ), RSV or RSV + IFN $\beta$. k IFN $\beta$ expression in AECs that were treated with murine recombinant IL-33 (IL-33), RSV, or RSV + IL-33. Data represents the Mean \pm SE from 4 replicates per group (experimental repeats $3-4) .{ }^{*} p \leq 0.05,{ }^{* *} p \leq .01,{ }^{* * *} p \leq 0.001$.

$\mathrm{nM}$ ) (Allopurinol, Sigma Aldrich) or with murine recombinant cytokines IL-1 $\beta$, IL-33, or IFN $\beta(10 \mu \mathrm{g} / \mathrm{ml})$. Uric acid crystals were prepared as described previously. ${ }^{31,32}$ Briefly, $0.03 \mathrm{M}$ solution of monosodium urate (MSU) or uric acid at a volume of $200 \mathrm{ml}$ was prepared by adding $1 \mathrm{~g}$ of uric acid crystalline (Sigma, St Louis, Missouri, USA) in a solution containing $6 \mathrm{ml}$ of $1 \mathrm{~N}$ sodium hydroxide $(\mathrm{NaOH})$, and adjusted $\mathrm{pH}$ to 7.2 with $\mathrm{HCl}$. The solution became pyogenic free after incubation for six hours at $120^{\circ} \mathrm{C}$.

Derivation of bone marrow-derived macrophages (BMDMs) BMDMs from single-cell suspensions of tibia and femur marrow were differentiated in vitro as previously described. ${ }^{33}$ Briefly, murine bone marrow was cultured in RPMI 1640 (Lonza, Walkersville, MD) supplemented with $30 \%$ L-cell conditioned media, 20\% fetal calf serum and penicillin/streptomycin for six days. Adherent BMDMs were harvested and replated in complete media. BMDMs were treated with XOI (200 nM) (Allopurinol, Sigma Aldrich) or with murine recombinant cytokine IL-33 $(10 \mu \mathrm{g} / \mathrm{ml})$. Uric acid crystals were prepared as described previously. The BMDMs were treated with $\mathrm{XOI}$, or IL-33, or uric acid alone or during RSV infection. Cultures were infected with RSV at 1:1 MOl, for $4 \mathrm{~h}$ for RNA measurement and $24 \mathrm{~h}$ for protein quantification in cell culture supernatants.

Human airway epithelial cells and RSV infection A549 cells (ATCC) were grown in Ham's F-12 medium containing 1 $\mathrm{mM}$ L-glutamine (Life Technologies) with 10\% FBS (Quality Biological, Gaithersburg, MD) and penicillin/streptomycin. 


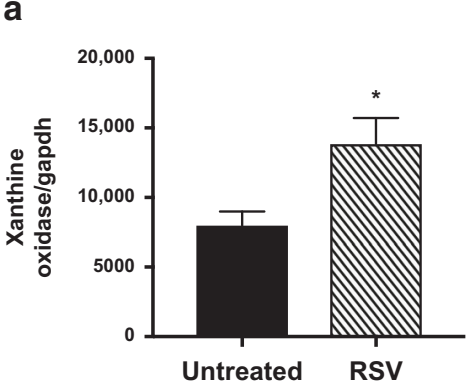

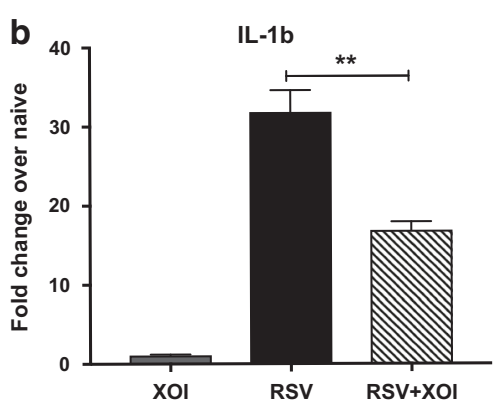

C

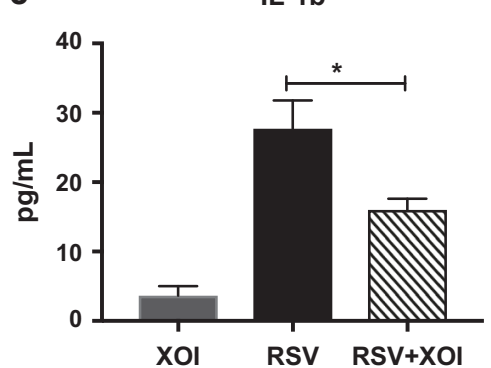

d

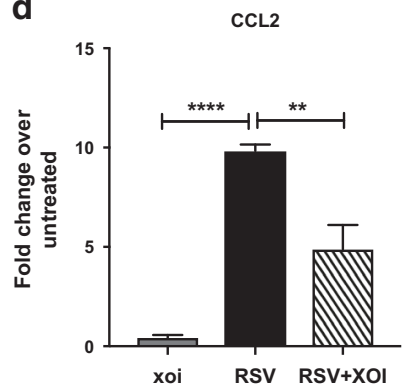

e

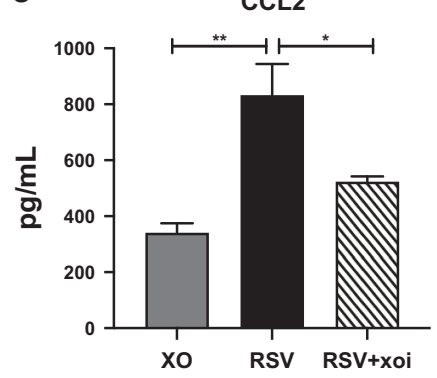

f

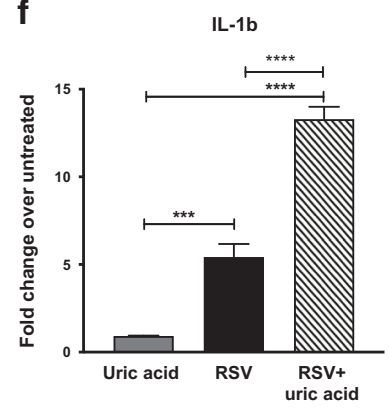

9

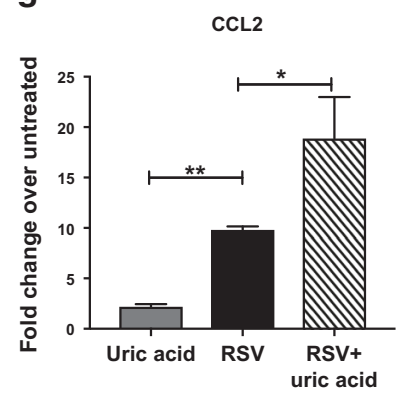

Fig. 5 Bone marrow-derived macrophages (BMDMs) treated with XOI during RSV infection show decreased expression of IL-1 $\beta$ and CCL2. a qPCR XO expression in BMDMs that were RSV infected. b, $\mathbf{c}$ Expression and production of IL- $\beta$ by BMDMs treated with or without XOI during RSV infection. $\mathbf{d}$, e CCL2 expression and production in BMDMs treated with or without XOI during RSV infection. $\mathbf{f}, \mathbf{g}$ Expression of IL-1 $\beta$ and CCL2 in BMDMs treated with uric acid during RSV infection. Data represent the Mean \pm SE from 4 replicates per group (experimental repeats 3). ${ }^{*} p \leq 0.05,{ }^{* *} p \leq .01,{ }^{* * *} p \leq 0.001$.

Normal human small epithelial cells ([SAEC], Lonza) were grown in Small airway epithelial cell growth medium ([SAEC-media], Lonza) containing SAEC supplements and growth factors ([SingleQuots], Lonza). The cells were maintained at $37^{\circ} \mathrm{C}, 5 \% \mathrm{CO}_{2}$. For the experiments, cells were seeded in 48 and 96 well plates and grown until $60-70 \%$ confluent. Cultures were infected with RSV at 1:1 MOI, for $24 \mathrm{~h}$ for RNA extraction.

\section{Statistical analysis}

Data were analyzed by Prism 6 (GraphPad Software). Data presented are mean values \pm SEM. Comparison of two groups was performed with an unpaired, two-tailed Student $t$-test. Comparisons of three or more groups were analyzed by ANOVA with a Tukey posttest. A $p$-value $<0.05$ was considered significant.

\section{RESULTS}

Uric acid metabolism in mice after RSV infection

Previous studies have shown that plasma metabolites correlated to the severity of the RSV immunopathology ${ }^{26}$ and our previous data indicated that IL-1 pathways are critical. ${ }^{17}$ In this work, we analyzed the expression of xanthine oxidase (XO), an inducer of the IL-1 inflammasome, which catalyzes the oxidation of hypoxanthine to xanthine and subsequently xanthine to uric acid. XO expression was measured in the lung of RSV infected mice at 2-days, 4-days, 6-days, and 8-days post-infection. Increased expression of XO began at day 2 and continued at day 6 and 8 post-RSV infection (Fig. 2a). Levels of uric acid in bronchoalveolar lavages (BAL) of RSV infected mice at 2-days, 4-days, 6-days, and 8days post-infection were assessed. We detected significant increased uric acid levels in the BAL of infected mice beginning at day 2 post-infection with a higher level of uric acid at day 6 and 8 (Fig. 2b). These data indicate that RSV infection upregulated xanthine oxidase and uric acid production in the lungs of infected mice. In order to demonstrate human patient relevance, we analyzed uric acid levels in samples of lung aspirates from ICU hospitalized infants that were positive for RSV infection by PCR analyses. Significantly increased levels of uric acid were detected in the samples from RSV infected children compared with aspirates from normal samples taken from uninfected infants hospitalized for scheduled surgery (Fig. 2c). Thus, uric acid levels and/or XO were significantly increased in pre-clinical modeling and clinical samples associated with RSV disease.

Airway epithelial cells increase the expression of XO during RSV infection and induce inflammatory innate cytokines

The primary cells that are infected by RSV to propagate the virus are airway epithelial cells. We determined whether uric acid pathways were upregulated during RSV infection in human airway epithelial cell line A549, which are permissive to RSV infection and express proinflammatory genes characteristic of the infection. ${ }^{34}$ We observed significant upregulated expression of XO in the RSV infected cells compared with uninfected group (Fig. 3a). When the cells were treated with a $\mathrm{XOI}$ at the time of infection significant decreased expression of IL-33, TSLP, and CCL2 compared with RSV infected cells was observed (Fig. 3b-d). Interestingly, treatment with XOI during RSV infection increased the expression of IFN $\beta$ compared with RSV infected cells (Fig. 3e). We also used a primary human small epithelial cells (SAEC), since immortalized cell lines may not represent an ideal model for the study of RSV entry as they differ in the expression of surface receptors with primary human airway cells. ${ }^{35-37}$ We infected SAEC with RSV and we observed increased XO expression after infection (Supplementary Fig. 1a) as well as with infection of rhinovirus (Supplementary Fig. 1b) consistent with increased uric acid production. We also detected decreased expression of IL-33 as well as increased expression of IFN $\beta$ after infection with RSV (Supplementary Fig. 1c, d), similar to what was observed in the A549 cell line.

To further investigate the mechanism by which uric acid pathways and IL-1 $\beta$ modulated RSV immunopathology we used primary airway epithelial cells (AEC) isolated from naïve mice. First, we observed significantly increased expression of XO from RSV 
a

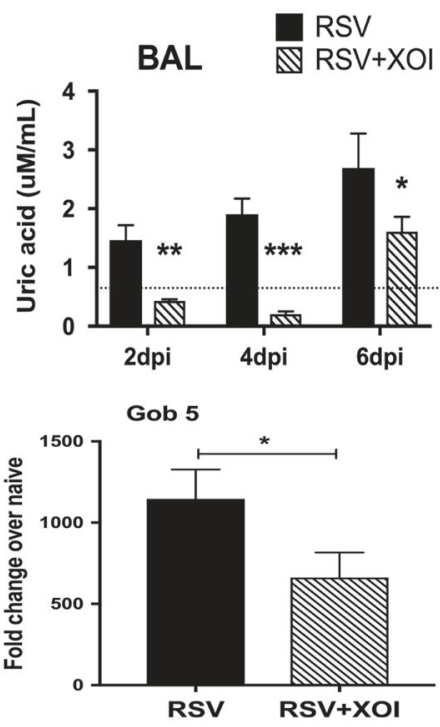

b
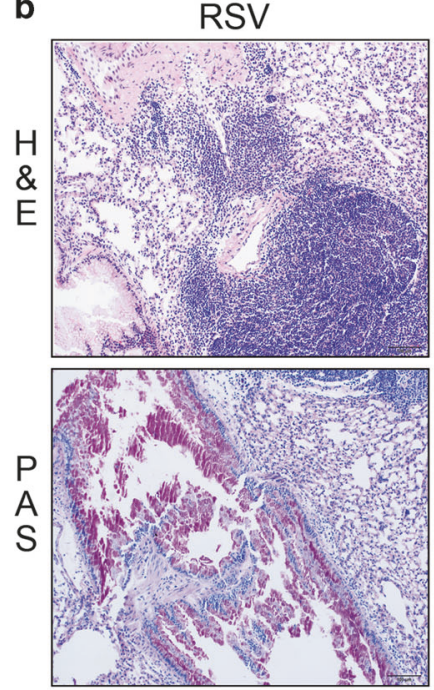

d

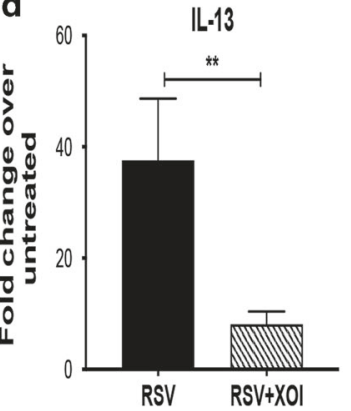

e

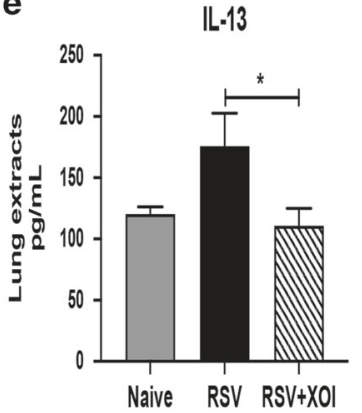

$\mathbf{f}$
LDLN
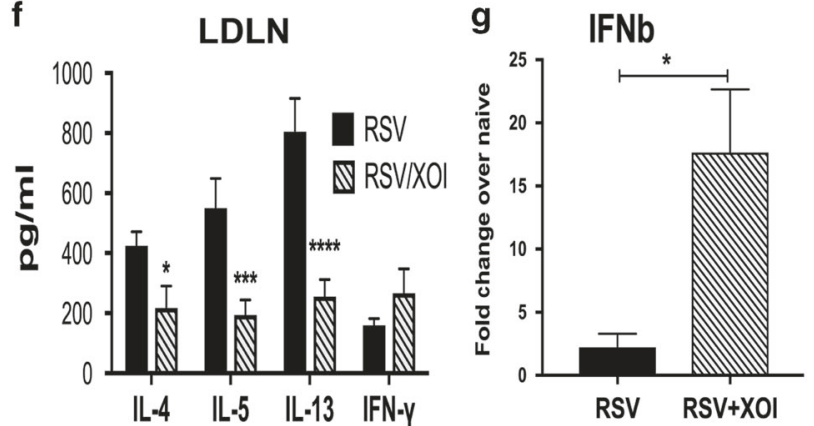

h

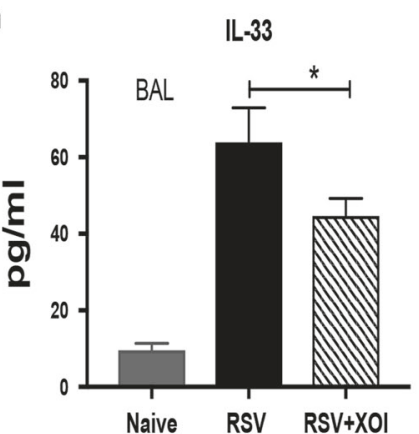

k

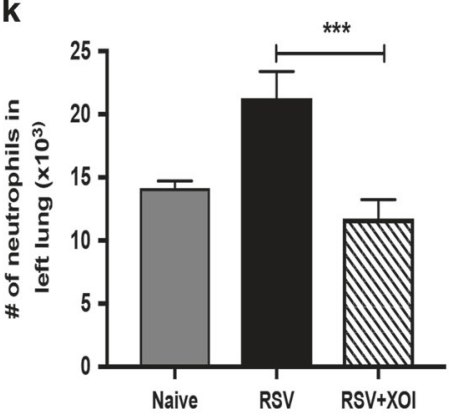

i

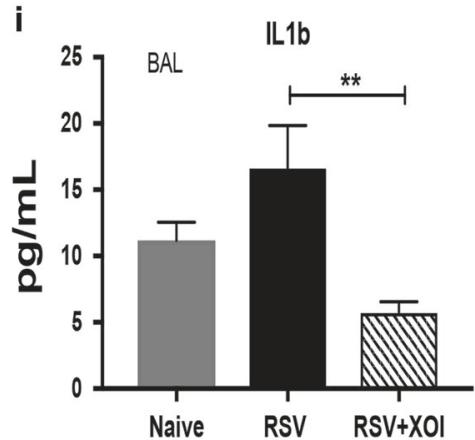

j

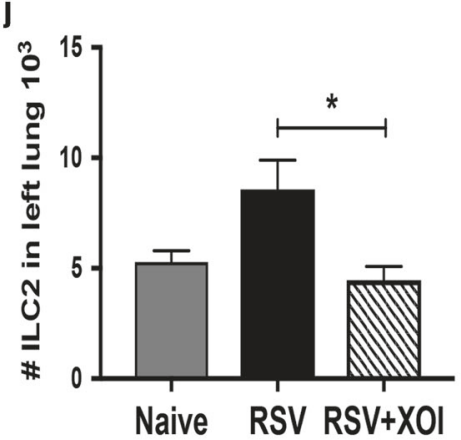

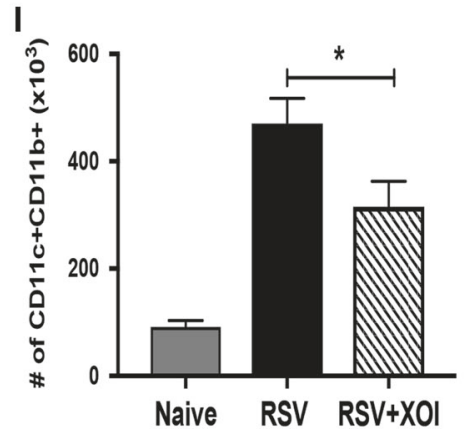

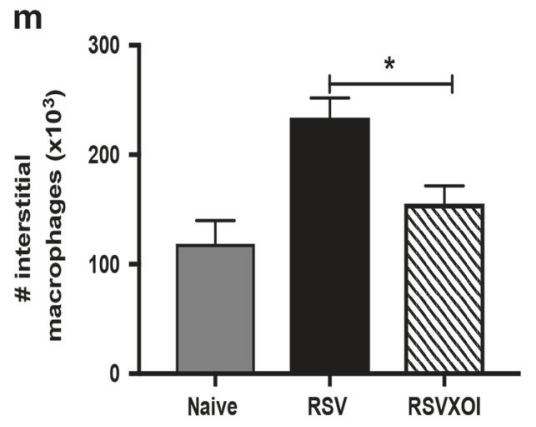

infected AEC (Fig. 4a). Next, we measured the expression of cytokines produced by AEC during RSV infection with or without $\mathrm{XOI}$ and observed inhibition of IL-33 and TSLP during RSV infection when the cells were treated with XOI (Fig. 4b). As with the A549 cells, we observed increased expression of IFN $\beta$ when the AEC were infected and treated with XOI (Fig. 4c). The treatment with the inhibitor decreased the expression and production of CCL2 in the RSV infected cells (Fig. 4d, e). Treatment 
Fig. 6 Targeting the xanthine/uric acid pathway during RSV infection attenuates the immunopathology response. a Uric acid detection in the BAL of infected mice was significantly decreased in mice treated with XOI compared with control and RSV infected mice. b Lung histopathology in Hematoxylin and Eosin stain (H\&E) showed inflammatory infiltrates and Periodic acid-Schiff stain (PAS) to detected mucus that was reduced in the lungs of mice treated with XOI, as well as c decreased Gob 5 mRNA expression. d, e IL-13 lung mRNA expression and production in lung extracts. $f$ Lung draining lymph nodes from mice at 8 were re-stimulated with RSV (MOI of 1, 48 h), and T-cell cytokine levels were measured by Bio-Plex. $\mathbf{g}$ qPCR of total lung RNA IFN $\beta$ mRNA expression. $\mathbf{h}$, i ELISA of IL-33 and IL-1 $\beta$ from BAL samples collected at 6 dpi. j-m Flow cytometry of lung leukocytes, ILC2, neutrophils, dendritic cells, and interstitial macrophages from naïve and infected mice at 8 dpi. Data represents the Mean \pm SE from 4 to 5 mice (experimental repeats $4-5$ ). ${ }^{*} p \leq 0.05,{ }^{* *} p \leq 0.01,{ }^{* * *} p \leq 0.001$.

of the AEC with uric acid alone and during infection significantly increased the expression of IL-33 and CCL2 compared with RSV infected cells (Fig. 4f, g). Next, we investigated the effect of IL-1 $\beta$ in AEC during RSV infection and observed a significantly increased expression of IL-33 and CCL2 when the cells were co-incubated with IL-1 $\beta$ and RSV compared with RSV alone (Fig. 4h, i). Finally, the treatment of AEC with IL-33 during RSV infection significantly decreased the expression of IFN $\beta$, and the opposite was observed when the cells were under the treatment of IFN $\beta$ during RSV infection with IL-33 significantly reduced (Fig. 4j, k). Together these data demonstrate that AEC are activated by the uric acid pathway after RSV infection and that inhibiting XO during RSV infection decreased the expression of critical innate cytokines IL33, TSLP, and CCL2. We also infected AEC with rhinovirus to evaluate the potential of other respiratory virus to increased uric acid, and we observed that rhinovirus also increased the expression of xanthine oxidase in AEC, suggesting that uric acid could be upregulated by this virus and it is not an RSV dependent effect (Supplementary Fig. 2a). Rhinovirus also upregulated the expression of TSLP and IL-33 (Supplementary Fig. 2b).

BMDMs treated with $\mathrm{XOI}$ during RSV infection showed decreased expression of IL-1 $\beta$ and CCL2

To study the effect of the uric acid pathway during RSV infection in macrophages, BMDMs were grown from naïve mice and treated with $\mathrm{XOI}$ or uric acid during the RSV infection. The expression of $X O$ in BMDMs that were RSV infected was significant increase in BMDMs compared with uninfected controls (Fig. 5a). In contrast, BMDMs treated with $\mathrm{XOI}$ during RSV infection had significantly decreased expression and production of IL-1 $\beta$ compared with infected controls (Fig. 5b, c). Similar to these results, decreased expression and production of CCL2 from BMDMs treated with XOI during infection was detected (Fig. 5d, e). Finally, when BMDMs were treated with uric acid during RSV infection significant increased expression of IL-1 $\beta$ and CCL2 compared with only RSV infected cells was observed (Fig. 5f, g). Thus, uric acid enhanced macrophage-derived innate cytokines, especially IL-1 that may be involved in exacerbated RSV-induced disease.

Targeting the xanthine/uric acid pathway during RSV infection altered the immunopathology response

To study the participation of the uric acid pathway during RSV infection Balb/c female mice were infected with RSV and treated with $\mathrm{XOI}$ intraperitoneally daily during the infection (depicted in Fig. 1). Uric acid levels were measured in the BAL of mice at 2-days, 4-days, and 6-days post-infection, and we observed that the mice that were treated with XOI had significantly decreased levels of uric acid compared with untreated RSV infected mice (Fig. 6a). The lung histology of treated mice demonstrated that XOI reduced the levels of inflammatory infiltrates and mucus in the airways (Fig. 6b). Quantifying mucus related gob5 gene by qPCR (Fig. 6c) showed significantly decreased expression in XOI compared to the control treated mice. The expression and production of IL-13 in the lung of $\mathrm{XOI}$ treated mice was also significantly decreased compared with the control of RSV infected mice (Fig. 6d, e). No difference was detected in the production of IFNY (SF3C). To examine the effects on the acquired immune response cytokines from RSV re-stimulated lung draining lymph nodes (LDLN) showed significantly decreased production of IL-4, IL-5, and IL-13 in XOI treated mice compared with controls (Fig. 6f). In contrast, the expression of IFN $\beta$ in the lungs of $\mathrm{XOI}$ treated mice was significantly upregulated (Fig. $6 \mathrm{~g}$ ), while they had significantly decreased IL-33 and IL-1 $\beta$ compared with control RSV infected mice (Fig. 6h, i). No difference was detected in the expression of IFNa or production of IFNy (Supplementary Fig. 3a-d). To characterize the cellular infiltration, total leukocyte numbers were examined in the lung by flow cytometry. The XOI treated mice had significant decreases in the numbers of ILC2, neutrophils, dendritic cells (DCs) and interstitial macrophages (Fig. 6j-m) compared to control treated mice. No difference was detected in the expression RSV F and G genes in the lung of the mice infected (Supplementary Fig. 3e, f). The allopurinol treated mice had no changes in behavior and no apparent developmental changes. Thus, by blocking the activity of xanthine oxidase and decreasing the production of uric acid in the lungs of RSV infected animals the pathology was controlled.

\section{Targeting IL-1 pathway during RSV infection altered the immunopathology response}

A primary downstream effect of uric acid is NLRP3 inflammasome pathway activation and production of IL-1. In order to determine the role of IL-1 mice were treated with interleukin-1 receptor antagonist (IL1-ra) intranasally during the infection (as depicted in Fig. 1). IL-1 ra binds to IL-1 receptors but does not induce any intracellular response and therefore acts as a receptor antagonist. ${ }^{38}$ The lung histology of IL-1ra treatment profoundly reduced the levels of inflammatory infiltrates and mucus deposition in the airways (Fig. 7a). We detected significantly decreased expression of gob5 gene (Fig. 7b) as well as significantly decreased expression and production of $\mathrm{IL}-13$ in the lungs of IL-1ra treated mice compared with the control mice (Fig. 7c, d), and significant decreased production of IL-5 in the group of treated mice (Fig. 7e). No difference was detected in the production of IFNY (Supplementary Fig. 3c). The production of cytokines from RSV restimulated lung draining lymph nodes (LDLN), showed significantly decreased production of IL-4 and IL-13 in IL-1ra treated mice compared with controls (Fig. 7f). IFN $\beta$ expression in the lung of IL1ra treated mice was significantly increased in comparison with control RSV infected mice (Fig. 7g). No difference was detected in the expression of IFNa (Supplementary Fig. 3b). We also detected decreased levels of IL-33 in BAL fluid of IL-1ra treated group compared with the control group (Fig. 7h). Total leukocyte numbers were examined in dispersed lungs, and significant decreases in the numbers of ILC2 cells, neutrophils, interstitial macrophages, dendritic cells, and T cells (CD4+, CD4+ $\mathrm{CD69+}$, and $\mathrm{CD} 8+$ ) were observed in the IL1-ra treated group compared with control RSV infected group (Fig. 7l-m). No difference was detected in the expression RSV $F$ and G genes in the lung of the mice infected (Supplementary Fig. 3d). Thus, similar to treating mice with a Xanthine Oxidase Inhibitor, blockade of IL-1 signaling appears to reduce pathologic responses.

\section{DISCUSSION}

The present study demonstrates that uric acid is upregulated during RSV infection and increased production of uric acid has a significant effect in RSV-immunopathology related to key 
a
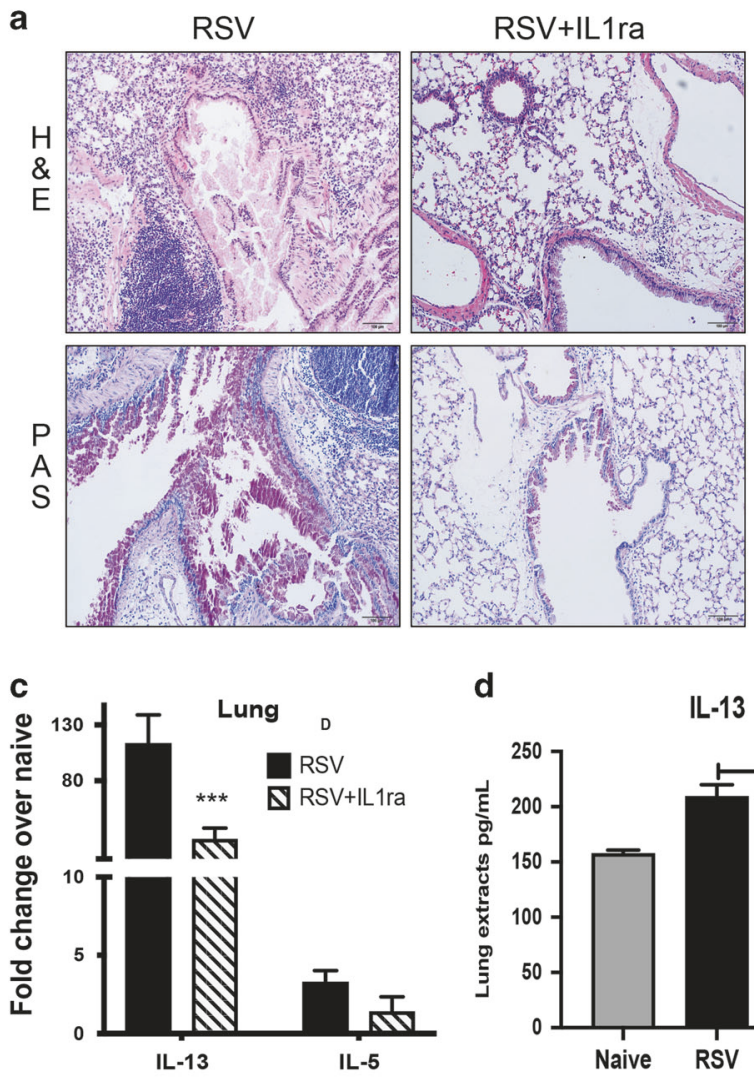

d

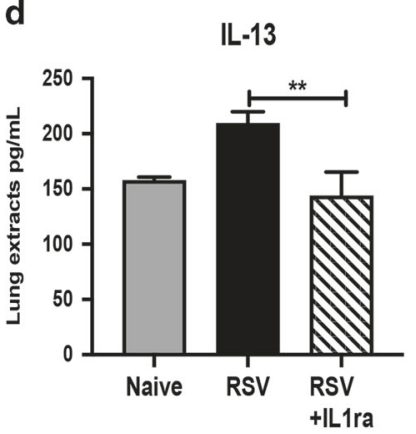

e

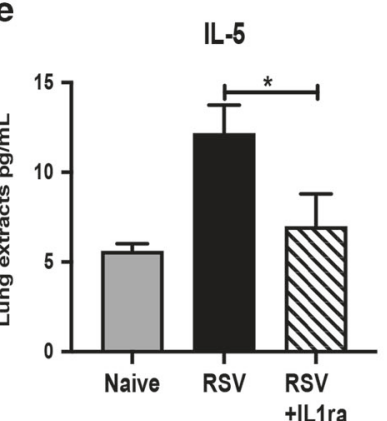

g

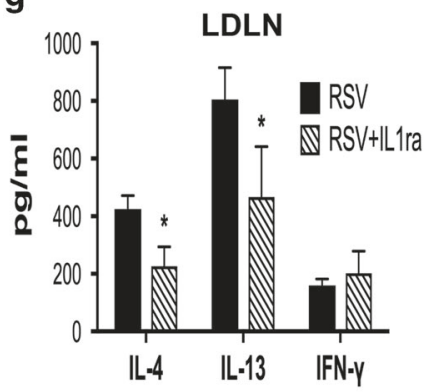

k

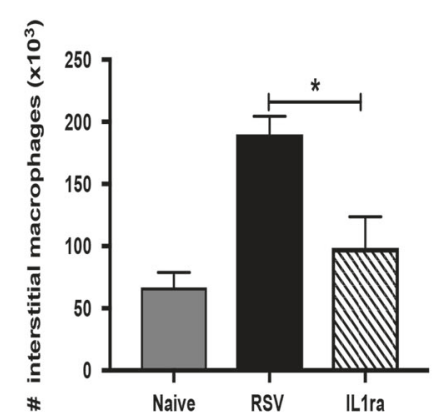

h

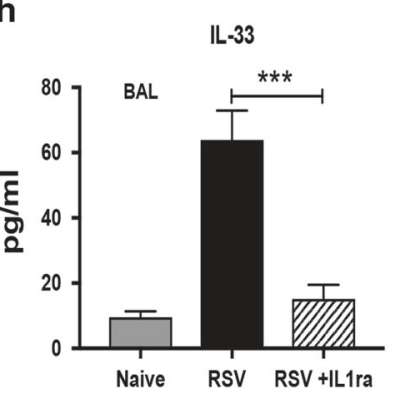

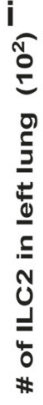

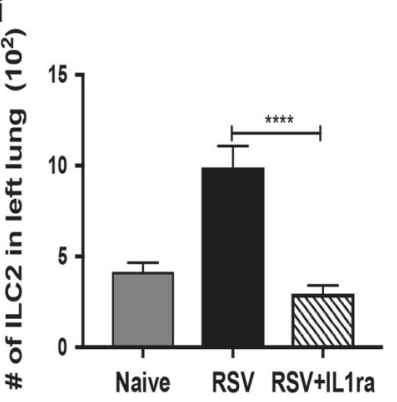

f

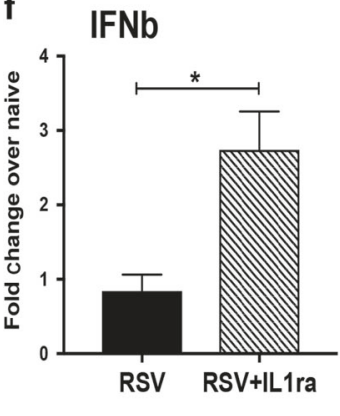

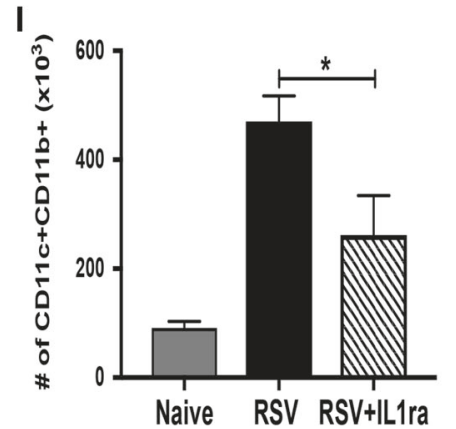

m
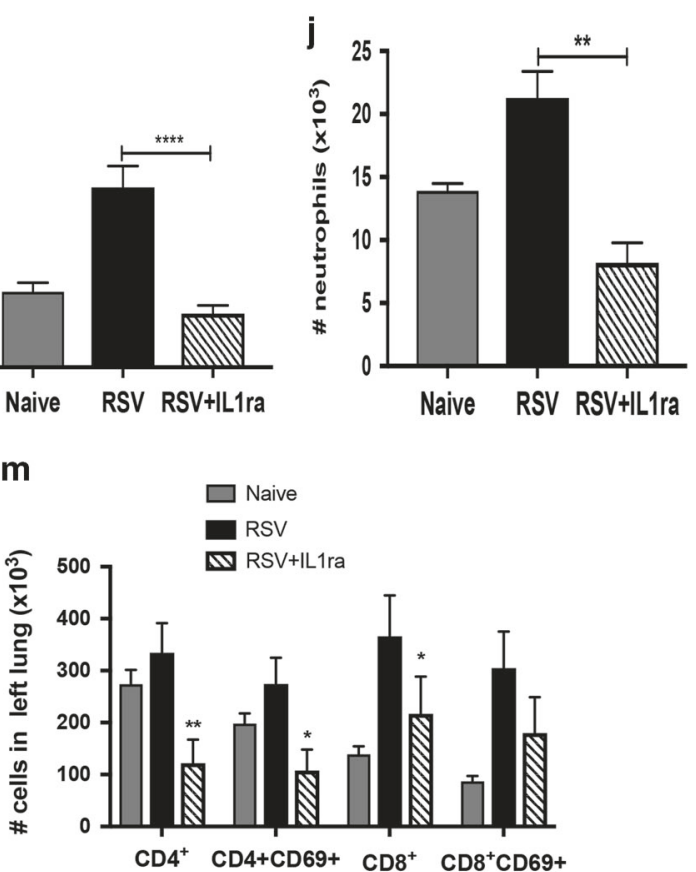

Fig. 7 Targeting IL-1 pathway during RSV infection attenuates the immunopathology response. a Lung histopathology (H\&E) showed inflammatory infiltrated and mucus (PAS staining) were reduced in lungs of mice with IL-1 ra as well as b decreased Gob 5 and Muc5 mRNA expression, and $\mathbf{c}$ IL-13 and IL-5 mRNA expression. $\mathbf{d}$, e IL-13 and IL-5 production in lung extracts. $\mathbf{f}$ qPCR of total lung RNA IFN $\beta$ expression. g Lung draining lymph nodes from mice at $8 \mathrm{dpi}$ were re-stimulated with RSV (MOI of 1, $48 \mathrm{~h}$ ), and T-cell cytokine levels were measured by Bio-Plex. h ELISA of IL-33 from BAL samples collected at $6 \mathrm{dpi}$. i-m Flow cytometry of lung leukocytes, ILC2 cells, neutrophils, interstitial macrophages, dendritic cells, and T cells from naïve and infected mice at $8 \mathrm{dpi}$. Data represents the Mean \pm SE from 4 to 5 mice (experimental repeats $3-4) .{ }^{*} p \leq 0.05,{ }^{* *} p \leq .01,{ }^{* * *} p \leq 0.001$. 


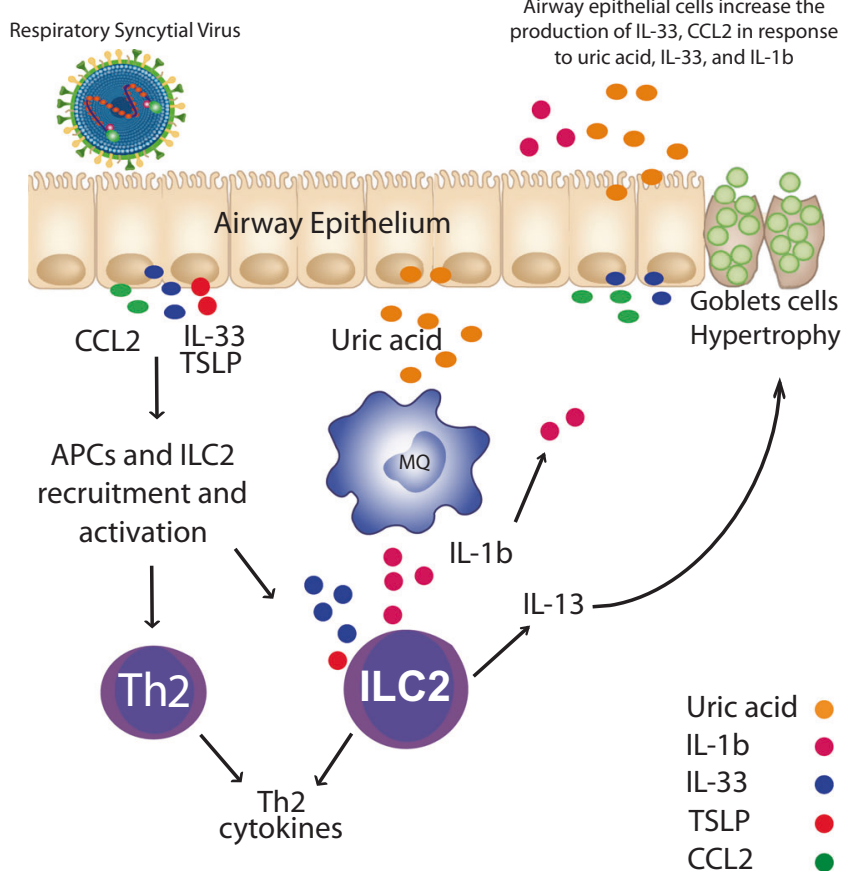

Fig. 8 RSV induces Uric acid and leads to Th2 response. RSV infected airway epithelial cells (AECs) trigger the production of uric acid, IL-33, TSLP, and CCL2. AECs and macrophages increase the expression of innate cytokines when uric acid is upregulated. CCL2 can recruit monocytes, APC, and T cells to the lung, while IL-33 along with TSLP and IL-1 $\beta$ will recruit and activate ILC2.

cytokines IL-1 $\beta$ and IL-33 in the lung. Importantly, these cytokines appear to be produced in response to uric acid from different cell populations, macrophages and epithelial cells, respectively. Our previous investigations have indicated how plasma metabolites can impact RSV-immunopathology by modulating metabolic pathways. $^{26}$ Here we described the role of uric acid, a final product of purine metabolism that is upregulated during RSV infection in infants as well as our mouse model and related to RSVimmunopathology. Uric acid is a danger associated molecular pattern (DAMP) that is secreted during cellular stress or by dying cells that can initiate and perpetuate a noninfectious inflammatory response. ${ }^{39}$ Uric acid crystals on their own are known to be the cause of gout; they trigger neutrophilic inflammation by activation of the NLRP3 inflammasome pathway leading to release of IL$1 \beta .^{19,20}$ The production of uric acid in human airway epithelial cells has been reported, and it is recognized that uric acid in the airways can act as a damage associated molecular pattern. ${ }^{40}$ Humans have a dysfunctional uricase and cannot degrade uric acid into hydrophilic allantoin, making them more vulnerable to variation of uric acid concentrations and susceptible to NRLP3 inflammasome activation-induced pathology. ${ }^{40,41}$ Together the present studies identify critical findings that indicate; (1) uric acid associated pathways may be a primary driver of RSV-induced pathology; (2) blocking either the production of uric acid or the downstream innate cytokine IL-1 activation pathways modulate the severity of infection; and (3) this mechanism appears to regulate critical cell populations, ILC2 and inflammatory DC, that can promote an altered immune environment.

Primary human airway epithelial cells from asthma, but not COPD patients, display elevated levels of extracellular uric acid in culture, ${ }^{40}$ suggesting that uric acid production is not generated in all diseases, rather it is upregulated in the presence of specific stimuli. A primary mechanism for uric acid induced inflammation is through the NLRP3 inflammasome pathway that drives IL-1 processing and inflammatory responses. Uric acid has also been described as an initiator and amplifier of Th2 responses independent of NLRP3/inflammasome/IL-1 pathway. ${ }^{42}$ The latter was previously demonstrated using a murine model in which uric acid drove Th2 response to OVA, where Wild-type C57BL/6 mice immunized with OVA + uric acid or HDM showed a marked increase in eosinophilia and lymphocytosis in BAL. This was also shown and maintained in Nlrp3 $3^{-1-}$ and Pycard ${ }^{-1-}$ demonstrating that uric acid can act independently of the inflammasome pathway to generate Th2 immunity. ${ }^{42,43}$ Important to disease, uric acid in the airways is increased after allergen-challenge in asthmatic patients and in mice. ${ }^{42}$ Our data are the first to show that increased uric acid production during RSV infection exacerbates Th2 responses. This Th2 enhancement may be through the activation of IL-33, TSLP44, and CCL2 in airway epithelial cells and by the production of $\mathrm{IL}-1 \beta$ by macrophages. Rhinovirus also increased the expression of xanthine oxidase in AEC suggesting that uric acid production could be influencing disease in respiratory viral infections in general. The coordinated production of innate cytokines from these different cell populations may further amplify the response since they exacerbate different pathways and together enhance ILC2 and inflammatory DC accumulation, activation that likely drive RSV-induced pathology. These findings are consistent with previous studies examining cell-specific expression of TSLP, IL-33, and CCL2 $2^{39,43-47}$ that leads to Th2 immune responses by subsequent ILC2 activation. ${ }^{9,14,48}$ Here we extend these findings to suggest a pathway by which RSV can trigger the expression of these innate cytokines by uric acidmediated mechanisms likely through the upregulation of purine metabolism. Interestingly, there appears to be an inverse relationship of IL-33 with type I IFN during RSV infection, both in vitro and in vivo, which was influenced by uric acid pathways. This latter regulation was directly correlated to both the Th2 cytokines as well as the immunopathology of the ongoing mucus responses.

The XOI and IL-1ra treated animals had a similar reduction in IL13 , mucus, and ILC2 numbers, as well as an overall reduction in Th2 immune response. The accompanying decrease in Th2 cytokines in lung draining lymph nodes could be due to the decreased ILC2 that are a primary source of Th2 responses during RSV infection. $9,14,49,50$ These data correspond to the reduced IL-33, TSLP, and IL-1 $\beta$ in the $\mathrm{XOI}$ and IL-1ra treated groups related to the recruitment and activation of ILC2. ${ }^{9,16,51-53}$ The use of isolated murine lung epithelial cells and macrophages further demonstrated that different cellular targets of uric acid and IL-1 contribute with different innate cytokines that promote the pathogenic responses. These findings provide mechanistic insight into the development of RSV immunopathology and denoted that UA is a key immunoregulator in the respiratory mucosa (Fig. 8). Thus, metabolic changes during RSV infection focused on purine metabolism/uric acid pathway leading to a number of detrimental pathological immune responses that can be modulated by XOI or by inhibiting the effect of IL-1 $\beta$ with IL-1ra (Anakinra). Both treatments have been extensively tested for safety and are FDA approved for both adults and children ${ }^{21-23,25}$ and could be considered for severe RSV infection and perhaps to prevent RSVenhanced asthma predisposition by altering the lung immune environment.

\section{ACKNOWLEDGEMENTS}

The manuscript was supported in part by NIH grants Al036302 (NWL) RO1HL144858, and Al138348 (NWL) and the Mary H. Weiser Food Allergy Center. We thank Judith Connett, Ph.D., for editorial review.

\section{AUTHOR CONTRIBUTIONS}

The experiments were designed by N.W.L. and W.F. Experiments were performed by W.F., C.M., C.S., A.R., S.M. and S.K. Manuscript was written by N.W.L. and W.F. Data analysis was performed by W.F., C.M., C.S., A.R. and N.W.L. All authors participated in editing the manuscript. 


\section{ADDITIONAL INFORMATION}

The online version of this article (https://doi.org/10.1038/s41385-020-0264-z) contains supplementary material, which is available to authorized users.

Competing interests: The authors declare no competing interests.

Publisher's note Springer Nature remains neutral with regard to jurisdictional claims in published maps and institutional affiliations.

\section{REFERENCES}

1. Heilman, C. A. From the National Institute of Allergy and Infectious Diseases and the World Health Organization. Respiratory syncytial and parainfluenza viruses. J. Infect. Dis. 161, 402-406 (1990).

2. Openshaw, P. J., Dean, G. S. \& Culley, F. J. Links between respiratory syncytial virus bronchiolitis and childhood asthma: clinical and research approaches. Pediatr. Infect. Dis. J. 22, S58-S64 (2003).

3. Becker, S., Reed, W., Henderson, F. W. \& Noah, T. L. RSV infection of human airway epithelial cells causes production of the beta-chemokine RANTES. Am. J. Physiol. 272, L512-L520 (1997).

4. Chin, J., Magoffin, R. L., Shearer, L. A., Schieble, J. H. \& Lennette, E. H. Field evaluation of a respiratory syncytial virus vaccine and a trivalent parainfluenza virus vaccine in a pediatric population. Am. J. Epidemiol. 89, 449-463 (1969).

5. Fonseca, W., Lukacs, N. W. \& Ptaschinski, C. Factors affecting the immunity to respiratory syncytial virus: from epigenetics to microbiome. Front. Immunol. 9, 226 (2018).

6. Fonseca, W. et al. A recombinant influenza virus vaccine expressing the $F$ protein of respiratory syncytial virus. Arch. Virol. 159, 1067-1077 (2014).

7. Sigurs, N., Bjarnason, R., Sigurbergsson, F. \& Kjellman, B. Respiratory syncytial virus bronchiolitis in infancy is an important risk factor for asthma and allergy at age 7. Am. J. Respir. Crit. Care Med. 161, 1501-1507 (2000).

8. Stein, R. T. et al. Respiratory syncytial virus in early life and risk of wheeze and allergy by age 13 years. Lancet 354, 541-545 (1999).

9. Stier, M. T. et al. Respiratory syncytial virus infection activates IL-13-producing group 2 innate lymphoid cells through thymic stromal lymphopoietin. J. Allergy Clin. Immunol. 138, 814-824 e811 (2016).

10. Johnson, J. E., Gonzales, R. A., Olson, S. J., Wright, P. F. \& Graham, B. S. The histopathology of fatal untreated human respiratory syncytial virus infection. Mod. Pathol. 20, 108-119 (2007).

11. Zhu, Z. et al. Pulmonary expression of interleukin-13 causes inflammation, mucus hypersecretion, subepithelial fibrosis, physiologic abnormalities, and eotaxin production. J. Clin. Invest. 103, 779-788 (1999).

12. Halim, T. Y., Krauss, R. H., Sun, A. C. \& Takei, F. Lung natural helper cells are a critical source of Th2 cell-type cytokines in protease allergen-induced airway inflammation. Immunity 36, 451-463 (2012)

13. Barlow, J. L. et al. Innate IL-13-producing nuocytes arise during allergic lung inflammation and contribute to airways hyperreactivity. J. Allergy Clin. Immunol. 129, 191-198 (2012).

14. Saravia, J. et al. Respiratory syncytial virus disease is mediated by age-variable IL33. PLoS Pathog. 11, e1005217 (2015).

15. Qi, F. et al. Macrophages produce IL-33 by activating MAPK signaling pathway during RSV infection. Mol. Immunol. 87, 284-292 (2017).

16. Ohne, Y. et al. IL-1 is a critical regulator of group 2 innate lymphoid cell function and plasticity. Nat. Immunol. 17, 646-655 (2016).

17. Reed, M., Morris, S. H., Owczarczyk, A. B. \& Lukacs, N. W. Deficiency of autophagy protein Map1-LC3b mediates IL-17-dependent lung pathology during respiratory viral infection via ER stress-associated IL-1. Mucosal Immunol. 8, 1118-1130 (2015).

18. Owczarczyk, A. B. et al. Sirtuin 1 regulates dendritic cell activation and autophagy during respiratory syncytial virus-induced immune responses. J. Immunol. 195, 1637-1646 (2015)

19. Chen, C. J. et al. MyD88-dependent IL-1 receptor signaling is essential for gouty inflammation stimulated by monosodium urate crystals. J. Clin. Invest. 116, 2262-2271 (2006)

20. Martinon, F., Petrilli, V., Mayor, A., Tardivel, A. \& Tschopp, J. Gout-associated uric acid crystals activate the NALP3 inflammasome. Nature 440, 237-241 (2006)

21. Strilchuk, L., Fogacci, F. \& Cicero, A. F. Safety and tolerability of available uratelowering drugs: a critical review. Expert Opin. Drug Saf. 18, 261-271 (2019).

22. Dalbeth, N., Merriman, T. R. \& Stamp, L. K. Gout. Lancet 388, 2039-2052 (2016)

23. Shekelle, P. G. et al. Management of gout: a systematic review in support of an American College of Physicians Clinical practice guideline. Ann. Intern. Med. 166, 37-51 (2017).

24. Cortes, J. et al. Control of plasma uric acid in adults at risk for tumor Lysis syndrome: efficacy and safety of rasburicase alone and rasburicase followed by allopurinol compared with allopurinol alone-results of a multicenter phase III study. J. Clin. Oncol. 28, 4207-4213 (2010).

25. Goldman, S. C. et al. A randomized comparison between rasburicase and allopurinol in children with lymphoma or leukemia at high risk for tumor lysis. Blood 97, 2998-3003 (2001)

26. Fonseca, W. et al. Lactobacillus johnsonii supplementation attenuates respiratory viral infection via metabolic reprogramming and immune cell modulation. Mucosal Immunol. 10, 1569-1580 (2017).

27. Lukacs, N. W. et al. Differential immune responses and pulmonary pathophysiology are induced by two different strains of respiratory syncytial virus. Am. J. Pathol. 169, 977-986 (2006).

28. Newcomb, D. C. et al. Phosphatidylinositol 3-kinase is required for rhinovirusinduced airway epithelial cell interleukin-8 expression. J. Biol. Chem. 280, 36952-36961 (2005).

29. Miller, A. L., Strieter, R. M., Gruber, A. D., Ho, S. B. \& Lukacs, N. W. CXCR2 regulates respiratory syncytial virus-induced airway hyperreactivity and mucus overproduction. J. Immunol. 170, 3348-3356 (2003).

30. Reed, M. et al. Autophagy-inducing protein beclin-1 in dendritic cells regulates CD4 $\mathrm{T}$ cell responses and disease severity during respiratory syncytial virus infection. J. Immunol. 191, 2526-2537 (2013).

31. Kohn, N. N., Hughes, R. E., Mc, C. D. Jr. \& Faires, J. S. The significance of calcium phosphate crystals in the synovial fluid of arthritic patients: the "pseudogout syndrome". II. Identification of crystals. Ann. Intern. Med. 56, 738-745 (1962).

32. Giamarellos-Bourboulis, E. J. et al. Crystals of monosodium urate monohydrate enhance lipopolysaccharide-induced release of interleukin 1 beta by mononuclear cells through a caspase 1-mediated process. Ann. Rheum. Dis. 68, 273-278 (2009).

33. Ishii, M. et al. CC chemokine receptor 4 modulates Toll-like receptor 9-mediated innate immunity and signaling. Eur. J. Immunol. 38, 2290-2302 (2008).

34. Hillyer $P$. et al. Differential responses by human respiratory epithelial cell lines to respiratory syncytial virus reflect distinct patterns of infection control. J. Virol. 2018; 92, e02202-17.

35. Johnson, S. M. et al. Respiratory syncytial virus uses CX3CR1 as a receptor on primary human airway epithelial cultures. PLoS Pathog. 11, e1005318 (2015).

36. Zhang, L., Peeples, M. E., Boucher, R. C., Collins, P. L. \& Pickles, R. J. Respiratory syncytial virus infection of human airway epithelial cells is polarized, specific to ciliated cells, and without obvious cytopathology. J. Virol. 76, 5654-5666 (2002).

37. Patel, D. A. et al. Interferon response and respiratory virus control are preserved in bronchial epithelial cells in asthma. J. Allergy Clin. Immunol. 134, 1402-1412 (2014).

38. Arend, W. P., Malyak, M., Guthridge, C. J. \& Gabay, C. Interleukin-1 receptor antagonist: role in biology. Annu. Rev. Immunol. 16, 27-55 (1998).

39. Kale, S. L., Agrawal, K., Gaur, S. N. \& Arora, N. Cockroach protease allergen induces allergic airway inflammation via epithelial cell activation. Sci. Rep. 7, 42341 (2017).

40. Huff, R. D. et al. Regulation of xanthine dehydrogensase gene expression and uric acid production in human airway epithelial cells. PLoS One 12, e0184260 (2017).

41. Watanabe, S. et al. Uric acid, hominoid evolution, and the pathogenesis of saltsensitivity. Hypertension 40, 355-360 (2002).

42. Kool, M. et al. An unexpected role for uric acid as an inducer of $\mathrm{T}$ helper 2 cell immunity to inhaled antigens and inflammatory mediator of allergic asthma. Immunity 34, 527-540 (2011).

43. Hara, K. et al. Airway uric acid is a sensor of inhaled protease allergens and initiates type 2 immune responses in respiratory mucosa. J. Immunol. 192, 4032-4042 (2014).

44. Malinczak, C. A. et al. Sex-associated TSLP-induced immune alterations following early-life RSV infection leads to enhanced allergic disease. Mucosal Immunol. 12, 969-979 (2019).

45. Grainger, R., McLaughlin, R. J., Harrison, A. A. \& Harper, J. L. Hyperuricaemia elevates circulating CCL2 levels and primes monocyte trafficking in subjects with inter-critical gout. Rheumatology 52, 1018-1021 (2013).

46. Liang, W. Y. et al. Uric acid promotes chemokine and adhesion molecule production in vascular endothelium via nuclear factor-kappa B signaling. Nutr. Metab. Cardiovasc Dis. 25, 187-194 (2015).

47. Malinczak, C. A. et al. Upregulation of H3K27 demethylase KDM6 during respiratory syncytial virus infection enhances proinflammatory responses and immunopathology. J. Immunol. 204, 159-168 (2020).

48. Lee, H. C. et al. Thymic stromal lymphopoietin is induced by respiratory syncytial virus-infected airway epithelial cells and promotes a type 2 response to infection. J. Allergy Clin. Immunol. 130, 1187-1196 e1185 (2012).

49. Wang, D. et al. Respiratory syncytial virus prevents the subsequent development of ovalbumin-induced allergic responses by inhibiting ILC2 via the IL-33/ST2 pathway. Immunotherapy 10, 1065-1076 (2018). 
Uric acid pathway activation during respiratory virus infection promotes... W Fonseca et al.

50. Stier, M. T. et al. STAT1 represses cytokine-producing group 2 and group 3 innate lymphoid cells during viral infection. J. Immunol. 199, 510-519 (2017).

51. Camelo, A. et al. IL-33, IL-25, and TSLP induce a distinct phenotypic and activation profile in human type 2 innate lymphoid cells. Blood Adv. 1, 577-589 (2017).
52. Han, M. et al. The innate cytokines IL-25, IL-33, and TSLP cooperate in the induction of type 2 innate lymphoid cell expansion and mucous metaplasia in rhinovirus-infected immature mice. J. Immunol. 199, 1308-1318 (2017).

53. Martinez-Gonzalez, I., Steer, C. A. \& Takei, F. Lung ILC2s link innate and adaptive responses in allergic inflammation. Trends Immunol. 36, 189-195 (2015). 